\title{
Frequency of Extended-Spectrum Beta-lactamases (ESBLs) in strains of Klebsiella and E. coli isolated from patients hospitalized in Yazd
}

Hengameh Zandi ${ }^{1}$, Seyed Mostafa Tabatabaei ${ }^{2}$, Fatemeh Ehsani ${ }^{2}$, Mojtaba Babaei Zarch ${ }^{3}$, Samira Doosthosseini ${ }^{4}$

${ }^{1}$ Ph.D. in Microbiology, Assistant Professor, Department of Microbiology, School of Medicine, Shahid Sadoughi University of Medical Sciences, Yazd, Iran

${ }^{2}$ Resident of General Surgery, School of Medicine, Shahid Sadoughi University of Medical Sciences, Yazd, Iran

${ }^{3}$ Medical Student, Student Research Committee, Shahid Sadoughi University of Medical Sciences, Yazd, Iran

${ }^{4}$ General Practitioner, Yazd, Iran

\section{Type of article: Original}

\begin{abstract}
Introduction: Frequency of extended-spectrum beta-lactamases (ESBLs) and its variants may vary in different geographical areas, as reports indicate their spread in some certain communities. The aim of this study was to determine the frequency of ESBLs in strains of Klebsiella and E. coli, isolated from patients hospitalized in teaching hospitals of Yazd.

Methods: This cross-sectional study was carried out on samples including E. coli and Klebsiella strains collected from laboratories of Shahid Sadoughi and Shahid Rahnemoun hospitals in Yazd, Iran in the period of 2011-2012. The colonies which were positive in lactose Eosin methylene-blue (EMB) medium were identified by biochemical methods, and 270 strains of Klebsiella and E. coli were isolated. Collected data and information were analyzed using Fisher's exact test and descriptive statistics such as mean in SPSS software, version 15, at a significant level of 0.05 .

Results: In this study, 270 samples were examined, including 152 samples of $E$. coli (56.3\%) and 118 samples of Klebsiella pneumonia (43.7\%). Among the 152 samples of E. coli, 45 strains (30\%) were producers of ESBLs. In addition, among the 118 samples of Klebsiella pneumonia, 44 strains (37.3\%) were producers of ESBLs. E. coli strains showed the most resistance to Cefotaxime (100\%), Ceftazidime (97.7\%), and Cefepime (75.5\%) respectively and Klebsiella strains showed the most resistance to Cefotaxime (100\%), Ceftazidime (100\%) and Cefepime (79.5\%), respectively.

Conclusion: Frequency of ESBLs in Klebsiella strains was higher than E. coli strains. No significant relationship was found between frequency of ESBLs and age or gender. In addition, E. coli strains showed the highest sensitivity to Imipenem, Amoxicillin/clavulanate, and Ciprofloxacin, while the highest antibiotic sensitivity of Klebsiella strains was shown to be to Piperacillin, Imipenem, and Amoxicillin/clavulanate.

Keywords: ESBLs, Klebsiella, E. coli, Resistance
\end{abstract}

\section{Introduction}

Infections, caused by beta-lactam-resistant organisms, has increased due to the production of numerous enzymes in recent years (1). Extended-spectrum beta-lactamases (ESBLs) are a group of beta-lactamases that are most often derived from mutation in the older beta-lactamases that alters the amino acid configuration around the active site of these $\beta$-lactamases (2). ESBL production by organisms such as Enterobacteriaceae has created challenges to clinical microbiologists and clinicians (3). The prevalence of antibiotic resistance among E. coli and Klebsiella pneumoniae has been increased markedly in recent years by production of ESBLs (4). E. coli and Klebsiella pneumonia are mostly ESBL producers (5). One of the ways of resistance in Gram-negative bacteria is the production of ESBLs. The family Enterobacteriaceae produce ESBLs resulting in resistance to antibiotics (6). Frequency of ESBLs and its

\section{Corresponding author:}

Dr. Seyed Mostafa Tabatabaei, School of Medicine, Shahid Sadoughi University of Medical Sciences, Yazd, Iran. Tel: +98.3538224000, Fax: +98.3538224000, Email: dr_m_tabatabai@yahoo.com

Received: July 21, 2016, Accepted: October 02, 2016, Published: February 2017

iThenticate screening: September 03, 2016, English editing: November 21, 2016, Quality control: January 04, 2017

(C) 2017 The Authors. This is an open access article under the terms of the Creative Commons Attribution-NonCommercialNoDerivs License, which permits use and distribution in any medium, provided the original work is properly cited, the use is non-commercial and no modifications or adaptations are made. 
variants may vary in different geographical areas, as reports indicate their spread in some certain communities. Resistance of organisms to face antibiotics has become a problem around the world with serious consequences on the treatment of infectious diseases (1). Hence, the aim of present study was to determine the frequency of ESBLs in strains of Klebsiella and E. coli isolated from patients hospitalized in teaching hospitals of Yazd in the period 2011 2012 .

\section{Material and Methods}

\subsection{Design and setting}

The present research was a cross-sectional, descriptive-analytic study. Using total enumeration method, all samples including E. coli and Klebsiella strains collected from laboratories of Shahid Sadoughi and Shahid Rahnemoun hospitals in Yazd Province were studied. Eosin methylene-blue (EMB) lactose sucrose agar was used for bacteria culture. EMB is a useful differential-selective medium for isolation and identification of Gram-negative intestinal bacteria. Eosin y and methylene blue are optional components added to prevent the growth of Gram-positive bacteria, while allowing the Gram-negative ones to grow. Lactose carbohydrates are also added to separate the isolates based on fermentation of lactose and sucrose. E. coli is a lactose-fermenting coliform which typically creates blue-black colonies with a greenish metallic luster. Other fermenting coliforms such as Enterobacter create pink colonies. Non-fermenting colonies are transparent, amber or colorless (7).

\subsection{Sample collection}

Gram-negative bacilli, isolated from different clinical specimens in laboratories of Shahid Sadoughi and Shahid Rahnemoun hospitals in Yazd Province were collected. After filling out the questionnaire (demographic information such as, name, age, gender, sample type, ward, and hospital and information on tests such as bacteria name, presence or absence of ESBLs, and antibiogram result), samples were transferred to the Microbiology Laboratory of Yazd University of Medical Sciences. Samples were cultured in EMB medium (Merck Company, Germany) and kept at a temperature of $37^{\circ} \mathrm{C}$ for 24 hours. The colonies which were positive in lactose EMB medium (those that created a deep pink color or metallic luster) were identified by biochemical methods and 270 strains of Klebsiella and E. coli were isolated.

\subsection{The tests used for the identification of bacteria}

Lactose fermentation (in the Triple Sugar Iron agar), gas production (in the Triple Sugar Iron agar), urea hydrolysis (in the urea agar), the use of citrate (in the Simon Citrate), movement (in the SH2-Indol-Motility medium), indole production (in the SH2-Indol-Motility medium), methyl red test (in the Methyl Red-Vegesproskaure broth), and VP wax test (in the Methyl Red-Vegesproskaure broth).

\subsection{Measurement of bacteria sensitivity to antibiotics}

Sensitivity of E. coli and Klebsiella isolates to antibiotics of Gentamicin, Ciprofloxacin, Cotrimoxazole, Clavulanate/amoxicillin, Imipenem, Piperacillin, Cefepime, Ceftazidime, and Cefotaxime was measured by the disk diffusion method (Kirby-Bauer) according to CLSI protocols, using bacterial suspension with a dilution equivalent to opacity of half pipe McFarland and the solid medium of Muller-Hinton (Merck Company, Germany). For this purpose, a bacterial suspension with a dilution equivalent to opacity of half pipe McFarland was prepared $\left(1.5 \times 10^{8}\right.$ bacteria). Then, the suspension was inoculated on the plates containing Müller-Hinton agar medium and cultured with GRASS method. Antibiotics disks were put on the medium using sterile forceps, as they had a distance of 20 $\mathrm{mm}$ from each other and $15 \mathrm{~mm}$ from the wall of plate. The plates were incubated for 18 hours at a temperature of $37^{\circ} \mathrm{C}$. After the incubation period, the diameter of areola related to lack of bacterial growth around the disc, was measured using a ruler (in $\mathrm{mm}$ ) and compared to the table provided in CLSI protocols and then sensitivity (s), intermediate (I), and resistant (R) were qualitatively reported. All tests were performed on the standard strain of E. coli ATCC25922 as the control.

\subsection{Studying the existence of ESBLs}

For this purpose, combination disk method and Cefotaxime (CTX: 30 $\mathrm{g}$ ), Cefotaxime-clavulanic acid (CTX

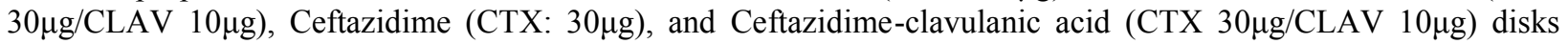
(Mast Company, England) were used. First of all, a bacterial suspension with a dilution equivalent to opacity of half pipe McFarland was prepared and then inoculated on the plates containing Müller-Hinton agar medium by a swab and cultured with GRASS method. The results were read after incubation. According to the manufacturer's instructions, if the diameter of areola related to lack of bacterial growth around the Ceftazidime-clavulanic acid or 
http://www.ephysician.ir

Cefotaxime-clavulanic acid disks shows an increase more than $5 \mathrm{~mm}$ compared to the diameter of areola related to lack of growth around Ceftazidime or Cefotaxime, this strain is considered as a producer of ESLBs.

\subsection{Data analysis}

Collected data and information were analyzed using descriptive statistics such as Fisher's exact test and mean in SPSS software, version 15 (SPSS Inc, Chicago, Illinois, USA), at a significant level of 0.05 .

\section{Results}

In this study, 270 samples were examined including 152 samples of E. coli $(56.3 \%)$ and 118 samples of Klebsiella pneumoniae (43.7\%). Among the 270 studied strains, 89 strains $(33 \%)$ were producers of ESBLs and 181 strains (67\%) were negative in terms of ESBLs production. Among the 152 samples of E. coli, 45 strains (30\%) were producers of ESBLs. In addition, among the 118 samples of Klebsiella pneumoniae, 44 strains (37.3\%) were producers of ESBLs (Table 1). According to the studies conducted on the frequency of ESBLs in strains of Klebsiella and E. coli in terms of gender, the following results were obtained: Among a total of 270 samples, 149 samples (55.1\%) male strains were cultured (73 strains of E. coli and 76 strains of Klebsiella). In this group, 21 samples of E. coli $(28.8 \%)$ and 28 samples of Klebsiella (36.8\%) were producers of ESLBs (p-value=0.3). Among a total of 270 samples, 121 samples (44.9\%) female strains were cultured (79 strains of E. coli and 42 strains of Klebsiella). In this group, 24 samples of E. coli (30.4\%) and 16 samples of Klebsiella (38.1\%) were producers of ESLBs (p-value $=0.42$ ) (Table 2). To determine the frequency of ESBLs in strains of Klebsiella and E. coli in terms of age, the studies samples were classified into four age groups including below 20, 20-40, 40-60, and above 60 . Among the 270 studied samples, 78 samples (28.8\%) were in the age group of 40-60 (44 strains of E. coli and 34 strains of Klebsiella). Among them, 9 samples of E. coli (20.4\%) and 11 samples of Klebsiella (32.3\%) were producers of ESLBs (p-value=0.29). In analysis of data on determining the frequency of ESBLs in strains of Klebsiella and E. coli in terms of sample type (Urine, ulcer, lung secretions, sputum, blood, and CSF), the following results were obtained: Among the 270 studied samples, 35 samples $(13 \%)$ were obtained from culture of ulcers $(15$ strains of E. coli and 20 strains of Klebsiella). Among them, 2 samples of E. coli (13.3\%) and 9 samples of Klebsiella (45\%) were positive in terms of ESLBs (p-value $=0.06$ ). Among the 270 studied samples, 7 samples $(2.59 \%)$ were obtained from culture of sputum ( 1 strain of E. coli and 7 strains of Klebsiella). Among them, only 2 samples of Klebsiella (33.3\%) were positive, in terms of ESLBs (p-value=1). Among the 270 studied samples, 20 samples (7.4\%) were obtained from culture of blood (12 strains of E. coli and 8 strains of Klebsiella). Among them, 7 samples of E. coli $(58.3 \%)$ and 3 samples of Klebsiella (37.5\%) were positive in terms of ESLBs (p-value=0.65). The results obtained from determining the resistance frequency of Klebsiella and E. coli strains to antibiotics of gentamicin, ciprofloxacin, cotrimoxazole, clavulanate/amoxicillin, imipenem, piperacillin, cefepime, ceftazidime, and cefotaxime by the diffusion disk method are as follows: Among the 89 samples producing ESBLs, 65 samples (73\%) $(68.8 \%$ of $E$. coli strains and $77.2 \%$ of Klebsiella strains) were resistant and 24 samples $(23 \%)(31.2 \%$ of $E$. coli strains and $22.8 \%$ of Klebsiella strains) were sensitive to cotrimoxazole (p-value $=0.47$ ). Among the 89 samples producing ESBLs, 52 samples (58.4\%) (71.1\% of E. coli strains and $45.4 \%$ of Klebsiella strains) were resistant and 37 samples (41.7\%) (28.9\% of E. coli strains and $54.6 \%$ of Klebsiella strains) were sensitive to piperacillin (pvalue $=0.01)$ (Table 3$)$.

Table 1. Determining the frequency ESBLs in strains of Klebsiella and E. coli using the combination disk method

\begin{tabular}{|l|l|l|l|}
\hline \multicolumn{2}{|l|}{ ESBLs } & $\mathrm{n}$ & $\%$ \\
\hline \multirow{3}{*}{ With } & E. coli & 45 & $30 \%$ \\
\cline { 2 - 4 } & Klebsiella & 44 & $37.3 \%$ \\
\cline { 2 - 4 } & All & 89 & $33 \%$ \\
\hline \multirow{3}{*}{ Without } & E. coli & 107 & $70 \%$ \\
\cline { 2 - 4 } & Klebsiella & 74 & $63.7 \%$ \\
\cline { 2 - 4 } & All & 181 & $67 \%$ \\
\hline
\end{tabular}

Table 2. Determining the frequency ESBLs in strains of Klebsiella and E. coli in terms of gender

\begin{tabular}{|c|c|c|c|c|c|c|c|c|c|c|c|c|c|c|}
\hline \multirow[t]{3}{*}{ Gender } & \multirow[t]{3}{*}{$\mathrm{n}$} & \multirow{2}{*}{\multicolumn{2}{|c|}{$\begin{array}{l}\text { With } \\
\text { enzyme }\end{array}$}} & \multirow{2}{*}{\multicolumn{2}{|c|}{$\begin{array}{l}\text { Without } \\
\text { enzyme }\end{array}$}} & \multicolumn{4}{|c|}{ E. coli } & \multicolumn{4}{|c|}{ Klebsiella } & \multirow{3}{*}{$\begin{array}{l}\mathrm{p}- \\
\text { value }\end{array}$} \\
\hline & & & & & & \multicolumn{2}{|c|}{$\begin{array}{l}\text { With } \\
\text { enzyme }\end{array}$} & \multicolumn{2}{|c|}{$\begin{array}{l}\text { Without } \\
\text { enzyme }\end{array}$} & \multicolumn{2}{|c|}{$\begin{array}{l}\text { With } \\
\text { enzyme }\end{array}$} & \multicolumn{2}{|c|}{$\begin{array}{l}\text { Without } \\
\text { enzyme }\end{array}$} & \\
\hline & & $\mathrm{n}$ & $\%$ & $\mathrm{n}$ & $\%$ & $\mathrm{n}$ & $\%$ & $\mathrm{n}$ & $\%$ & $\mathrm{n}$ & $\%$ & $\mathrm{n}$ & $\%$ & \\
\hline Male & 149 & 49 & 32.9 & 100 & 67.1 & 21 & 28.8 & 52 & 71.2 & 28 & 36.8 & 48 & 63.2 & 0.3 \\
\hline Female & 121 & 40 & 33.1 & 81 & 66.9 & 24 & 30.4 & 55 & 69.6 & 16 & 38.1 & 26 & 61.9 & 0.42 \\
\hline Total & 270 & 89 & 33 & 181 & 67 & 45 & 30 & 107 & 70 & 44 & 37.3 & 74 & 63.7 & - \\
\hline
\end{tabular}


Table 3. Determining the resistance frequency of Klebsiella and E. coli strains to different antibiotics by the diffusion disk method

\begin{tabular}{|l|l|l|l|l|l|l|l|l|l|}
\hline Antibiotic & \multicolumn{2}{|l|}{$\begin{array}{l}\text { Resistant to } \\
\text { antibiotic }\end{array}$} & \multicolumn{2}{l|}{$\begin{array}{l}\text { Sensitive to } \\
\text { antibiotic }\end{array}$} & \multicolumn{2}{l|}{ E. coli } & \multicolumn{2}{l|}{ Klebsiella } \\
\cline { 2 - 11 } & $\mathrm{n}$ & $\%$ & $\mathrm{n}$ & $\%$ & $\begin{array}{l}\text { \% of } \\
\text { resistance }\end{array}$ & $\begin{array}{l}\text { \% of } \\
\text { sensitive }\end{array}$ & $\begin{array}{l}\text { \% of } \\
\text { resistance }\end{array}$ & $\begin{array}{l}\text { \% of } \\
\text { sensitive }\end{array}$ & \\
\hline Gentamicin & 67 & 75.3 & 22 & 24.7 & 73.3 & 26.7 & 77.2 & 22.8 & 0.8 \\
\hline Cotrimoxazole & 65 & 73 & 24 & 23 & 68.8 & 31.2 & 77.2 & 22.8 & 0.47 \\
\hline Ciprofloxacin & 48 & 53.9 & 41 & 46.1 & 53.3 & 46.7 & 54.5 & 45.5 & 1 \\
\hline Piperacillin & 52 & 58.4 & 37 & 41.6 & 71.1 & 28.9 & 45.4 & 54.6 & 0.01 \\
\hline Cefotaxime & 89 & 100 & 0 & 0 & 100 & 0 & 100 & 0 & 1 \\
\hline Cefepime & 69 & 77.5 & 20 & 22.5 & 75.5 & 24.5 & 79.5 & 20.5 & 0.8 \\
\hline $\begin{array}{l}\text { Amoxicillin/ } \\
\text { clavulanate }\end{array}$ & 44 & 49.4 & 45 & 50.6 & 48.8 & 49.6 & 50 & 50 & 1 \\
\hline Ceftazidime & 88 & 98.9 & 1 & 1.1 & 97.7 & 0.3 & 100 & 0 & 1 \\
\hline Imipenem & 37 & 41.6 & 52 & 58.4 & 35.5 & 64.5 & 47.7 & 52.3 & 0.28 \\
\hline
\end{tabular}

\section{Discussion}

Since the 1980s, a new group of antibiotics called oxyimino cephalosporins, which were resistant to hydrolytic activity of beta-lactamase, were used for treatment of infections caused by Gram-negative bacteria $(8,9)$. In early 1990, ESBLs produced by Gram-negative bacteria, showed resistance to cephalosporins such as ceftazidime and cefotaxime, with higher resistance to cefotaxime than ceftazidime (10). ESBLs enzyme gene is related to plasmid and causes this enzyme to be easily transmitted among bacteria. In addition to the gene of ESBLs, most of these plasmids also transmit the genes resistant to several beta lactam and non-beta-lactam antibiotics (including aminoglycosides, cotrimoxazoles, and fluoroquinolones). As a result, most of isolated ESBLs are resistant to many types of antibiotics (11). In the present study, among the 154 strains of E. coli, 45 samples (30\%) were producers of ESBLs, while this figure in strains of Klebsiella was 44 out of 118 sample (37.3\%). Navarro conducted a study on the frequency of $E$. coli producing ESBLs, and the results showed that production of ESBLs in nosocomial samples of $E$. coli is $30 \%$ (12). In another study conducted by Ibrahim (2011) on the frequency of ESBLs in E. coli samples in Sudan, among the 233 samples of E. coli, 70 samples (30.2\%) were positive in terms of ESBLs (13). Eksi et al. (2007) studied 87 samples of E. coli of which 25 of them (32.1\%) showed production of ESBLs (14). The results of these studies on the frequency of ESBLs in E. coli strains are consistent with the findings of the present study with slight differences. In a study by Romero and two other studies carried out in Turkey, frequency of these enzymes in E. coli has been reported to be $1 \%, 12 \%$, and $19.5 \%$, respectively (15-17). These figures are less than those obtained in the present study. In studies conducted by Ghafourian, Korten in Turkey and Ozgunes, frequency of ESBLs in Klebsiella samples was reported to be $59.2 \%, 48.7 \%$, and $47 \%$, respectively. These figures are higher than the findings of the present study $(15,17,18)$. In addition, in studies carried out by Romero, prevalence of these enzymes was found to be $2.8 \%$ which is less than the figures in this study (16). As it can be seen in different studies, the frequency of ESBLs enzymes may vary in different regions, cities, and hospitals. In a study conducted in Thailand on various species of Enterobacteriaceae, no statistically significant association was found between age and ESBLs production in bacteria (6). In another study conducted by Ibrahim et al. on the frequency of ESBLs in E. coli samples in hospitals of Khartoum, Sudan from April to August 2011, no significant difference was observed between samples taken from children and adults in terms of ESBLs production (13). In the present study, according to the results obtained for age ranges (below 20, 20-40, 40-60, and above 60), no significant relationship was found between age ranges and studied samples ( $\mathrm{p}$-value $=1,0.54,0.29$, and 0.36 , respectively). In a study conducted by Luvsansharav et al. on different species of Enterobacteriaceae, E. coli was the most common species producing ESBLs and no statistically significant association was found between gender and ESBL production in bacteria (6). The relationship between gender and frequency of ESBLs in the present study was not significant, with p-values of 0.3 for men and 0.42 for women. As mentioned in the results, frequency of ESBLs in E. coli and Klebsiella strains in urine, blood, ulcer, CSF, lung secretions, and sputum was $27 \%$ and $31.5 \%, 58.3 \%$ and $37.5 \%, 13.3 \%$ and $45 \%$, $40 \%$ and $0 \%, 50 \%$ and $46 \%$, and $0 \%$ and $33.3 \%$, respectively. The highest frequency of these enzymes in E. coli and Klebsiella strains was obtained in blood culture and lung secretions culture, respectively. None of the findings for frequency of ESBLs in E. coli and Klebsiella strains in different samples were statistically significant. In a study in three hospitals in 3 regions of Iran, 113 samples of Klebsiella pneumoniae were isolated from respiratory tract infection that 67 samples (59.2\%) were producers of ESBLs (18). Accordingly, 17 samples in a hospital in Ilam 
(43.6\%), 37 samples in Milad Hospital of Tehran (74\%), and 13 samples in Imam Reza Hospital of Mashhad (54.27\%) were positive in terms of ESBLs (18). In the present study, frequency of ESBLs in Klebsiella samples of lung secretions culture was more than that of the hospital in Ilam but less than that of Milad Hospital of Tehran and Imam Reza Hospital of Mashhad. In another study in hospitals of Khartoum, Sudan, among the 233 samples of $E$. coli, 70 samples (30.2\%) were positive in terms of ESBLs. Most of these samples were related to ulcer culture (13). However, the highest frequency of ESBLs in the present study, was observed in blood culture samples. In the present study, $73.3 \%$ of E. coli and $77.2 \%$ of Klebsiella strains producing ESBLs were resistant to Gentamicin. These figures for resistance of E. coli and Klebsiella strains to cotrimoxazole, ciprofloxacin, piperacillin, cefotaxime, cefepime, amoxicillin / clavulanate, ceftazidime, and imipenem were $68.8 \%$ and $77.2 \%, 53.3 \%$ and $54.5 \%, 71.1 \%$ and $45.4 \%, 100 \%$ and $100 \%, 75.5 \%$ and $79.5 \%, 48.8 \%$ and $50 \%, 97.7 \%$ and $100 \%$, and $35.5 \%$ and $47.7 \%$, respectively. In the present study, resistance of Klebsiella to all antibiotics except piperacillin was higher than E. coli. Strains of E. coli and Klebsiella both showed the highest resistance to cefotaxime, ceftazidime, and cefepime. E. coli strains had the highest sensitivity to imipenem, amoxicillin/clavulanate, and ciprofloxacin, while Klebsiella strains showed the highest sensitivity to piperacillin, imipenem, and amoxicillin/clavulanate. Among antibiotics, only piperacillin, with a p-value of 0.01 , was statistically significant. In another study, 87 samples of $E$. coli and 40 samples of Klebsiella. ESBLs activity was measured by using the double synergism disk method. Activity of ESBLs was confirmed in 25 samples of E. coli (32.1\%) and 18 samples of Klebsiella (45\%). Resistance of ESBLs to ciprofloxacin, trimethoprim/sulfamethoxazole, gentamicin, and piperacillin/tazobactam in Klebsiella and $E$. coli samples was $56 \%$ and $76 \%, 55.6 \%$ and $68 \%, 64 \%$ and $77.8 \%$, and $28 \%$ and $50 \%$, respectively (14). These figures indicate higher resistance of E. coli than Klebsiella, while Klebsiella showed higher resistance than E. coli in the present study. The highest and lowest resistance in both bacteria, was shown to be related to gentamicin and piperacillin, respectively. In the present study, both E. coli and Klebsiella showed the highest resistance to gentamicin, while the lowest resistance of Klebsiella and E. coli was to piperacillin and ciprofloxacin, respectively. In a study on the frequency of ESBLs in E. coli samples in Sudan, among the 233 samples of E. coli, 70 samples $(30.2 \%)$ were positive in terms of ESBLs. According to the survey conducted on antibiotic resistance in strains producing ESBLs, $98.6 \%$ of them were resistant to cotrimoxazole and $81.4 \%$ showed resistance to ciprofloxacin (13). In the present study, percentage of resistance to above-mentioned antibiotics (except for cefotaxime which was completely consistent with the results of this study) was higher, especially in terms of imipenem and gentamicin. In addition, the highest and the lowest antibiotic resistance in the present study were related to cefotaxime and imipenem, respectively.

\section{Conclusions}

According to the study findings, frequency of ESBLs in Klebsiella strains was higher than E. coli strains. No significant relationship was found between frequency of ESBLs and age or gender. The highest frequency of ESBLs in E. coli and Klebsiella strains was found in blood culture and lung secretions culture samples, respectively. However, the findings showed no significant difference between E. coli and Klebsiella strains in terms of the frequency of ESBLs. In the present study, resistance of Klebsiella to all antibiotics except piperacillin was higher than E. coli. The highest antibiotic resistance in E. coli and Klebsiella was against cefotaxime and ceftazidime. In addition, E. coli strains showed the highest sensitivity to Imipenem, amoxicillin/clavulanate, and ciprofloxacin, while the highest antibiotic sensitivity of Klebsiella strains was shown to be to piperacillin, imipenem, and amoxicillin/clavulanate.

\section{Acknowledgments:}

The authors would like to thank Shahid Sadoughi University of Medical Sciences for supporting this study financially. The authors also thank the staff of Shahid Sadoughi and Shahid Rahnemoun hospitals.

\section{Conflict of Interest:}

There is no conflict of interest to be declared.

\section{Authors' contributions:}

All authors contributed to this project and article equally. All authors read and approved the final manuscript.

\section{References:}

1) Shaikh S, Fatima J, Shakil S, Rizvi SM, Kamal MA. Antibiotic resistance and extended spectrum betalactamases: types, epidemiology and treatment. Saudi J Biol Sci. 2015; 22(1): 90-101. doi: 10.1016/j.sjbs.2014.08.002. PMID: 25561890, PMCID: PMC4281622. 
2) Paterson DL, Bonomo RA. Extended-spectrum $\beta$-lactamases: a clinical update. Clin Microbiol Rev. 2005; 18(4): 657-86. doi: 10.1128/CMR.18.4.657-686.2005. PMID: 16223952, PMCID: PMC1265908.

3) Rupp ME, Fey PD. Extended spectrum beta-lactamase (ESBL)-producing Enterobacteriaceae: considerations for diagnosis, prevention and drug treatment. Drugs. 2003; 63(4): 353-65. doi: 10.1093/cid/civ333. PMID: 12558458.

4) Lautenbach E, Patel JB, Bilker WB, Edelstein PH, Fishman NO. Extended-spectrum beta-lactamaseproducing Escherichia coli and Klebsiella pneumoniae: risk factors for infection and impact of resistance on outcomes. Clin Infect Dis. 2001; 32(8): 1162-71. doi: 10.1086/319757. PMID: 11283805.

5) Taha S, Youssef N, Elkazaz A, Ramadan H. Detection of extended-spectrum beta-lactamases (ESBLs) in clinical isolates of Klebsiella pneumoniae using the ESBL NDP test and flow cytometric assay in comparison to the standard disc diffusion. Afr J Microbiol Res. 2015; 9(34): 1947-53. doi: 10.5897/AJMR2015.7691.

6) Luvsansharav UO, Hirai I, Niki M, Sasaki T, Makimoto K, Komalamisra C, et al. Analysis of risk factors for a high prevalence of extended-spectrum \{beta\}-lactamase-producing Enterobacteriaceae in asymptomatic individuals in rural Thailand. J Med Microbiol. 2011; 60(Pt5): 619-24. doi: 10.1099/jmm.0.026955-0. PMID: 21292857.

7) Winn W, Allen S, Jandana W. Koneman's Color atlas and textbook of diagnostic microbiology, 6th edition, Lippincott Williams and Wilkins. 2006; Appendix II.

8) Murray PR, Rosenthal KS, tobayashi GS, Pfaller MA. Medical Microbiology, 4th edition. Mosby Inc. 2009: 186.

9) Bradford PA. Extended-spectrum beta-lactamases in the 21st century: characterization, epidemiology, and detection of this important resistance threat. Clin Microbiol Rev. 2001; 14(4): 933-51. table of contents. doi: 10.1128/CMR.14.4.933-951.2001. PMID: 11585791, PMCID: PMC89009.

10) Hosoglu S, Gundes S, Kolayli F, Karadenizli A, Demirdag K, Gunaydin M, et al. Extended-spectrum betalactamases in ceftazidime-resistant Escherichia coli and Klebsiella pneumoniae isolates in Turkish hospitals. Indian J Med Microbiol. 2007; 25(4): 346-50. doi: 10.4103/0255-0857.37336. PMID: 18087082.

11) Todar K, Todar`s online text book of bacteriology. 2011. Available From: http://www.text book of bacteriology.net.

12) Navarro-Navarro M, Robles-Zepeda RE, Garibay-Escobar A, Ruiz-Bustos E. [Hospital and communityacquired beta-lactamases-producing Escherichia coli and Klebsiella pneumoniae at hospitals in Hermosillo, Sonora]. Salud Publica Mex. 2011; 53(4): 341-4. doi: 10.1590/S0036-36342011000400009. PMID: 21986791.

13) Ibrahim ME, Bilal NE, Magzoub MA, Hamid ME. Prevalence of Extended-spectrum beta-Lactamasesproducing Escherichia coli from Hospitals in Khartoum State, Sudan. Oman Med J. 2013; 28(2): 116-20. doi: 10.5001/omj.2013.30. PMID: 23599880, PMCID: PMC3628198.

14) Eksi F, Ozer G, Balci I. [Investigation of the frequency of extended spectrum beta-lactamases and antibiotic resistance in clinical isolates of Escherichia coli and Klebsiella sp]. Mikrobiyol Bul. 2007; 41(3): 447-52. PMID: 17933257.

15) Ozgunes I, Erben N, Kiremitci A, Kartal ED, Durmaz G, Colak H, et al. The prevalence of extendedspectrum beta lactamase-producing Escherichia coli and Klebsiella pneumoniae in clinical isolates and risk factors. Saudi Med J. 2006; 27(5): 608-12. PMID: 16680246.

16) Romero ED, Padilla TP, Hernandez AH, Grande RP, Vazquez MF, Garcia IG, et al. Prevalence of clinical isolates of Escherichia coli and Klebsiella spp. producing multiple extended-spectrum beta-lactamases. Diagn Microbiol Infect Dis. 2007; 59(4): 433-7. doi: 10.1016/j.diagmicrobio.2007.06.007. PMID: 17913435.

17) Korten V, Ulusoy S, Zarakolu P, Mete B, Turkish MSG. Antibiotic resistance surveillance over a 4-year period (2000-2003) in Turkey: results of the MYSTIC Program. Diagn Microbiol Infect Dis. 2007; 59(4): 453-7. doi: 10.1016/j.diagmicrobio.2007.06.016. PMID: 17888609 .

18) Ghafourian S, Bin Sekawi Z, Sadeghifard N, Mohebi R, Kumari Neela V, Maleki A, et al. The Prevalence of ESBLs Producing Klebsiella pneumoniae Isolates in Some Major Hospitals, Iran. Open Microbiol J. 2011; 5: 91-5. doi: 10.2174/1874285801105010091. PMID: 21915229 , PMCID: PMC3170933. 